LTH-856

\title{
Interpolations Among NAHE-based Supersymmetric and Nonsupersymmetric String Vacua
}

\author{
Alon E. Faraggi 母 $_{\text {and }}$ Mirian Tsulaia \\ Department of Mathematical Sciences, University of Liverpool, \\ Liverpool L69 7ZL, United Kingdom
}

\begin{abstract}
The quasi-realistic free fermionic heterotic-string models provide some of the most detailed examples to explore the phenomenology of string unification. While providing a powerful tool to generate models and their spectra, understanding the realisation of the free fermion models in a bosonic formalism will provide important insight into their basic properties away from the free fermion point. In this paper we elucidate bosonic equivalent of the basic symmetry breaking pattern in the free fermion models from $E_{8} \times E_{8}$ to $S O(16) \times S O(16)$ and exhibit the connection of the free fermion models with corresponding non-supersymmetric vacua by interpolations.
\end{abstract}

\footnotetext{
* E-mail address: faraggi@amtp.liv.ac.uk

ฯ E-mail address: tsulaia@liv.ac.uk
} 


\section{Introduction}

The heterotic-string models in the free fermionic formulation are among the most realistic string models constructed to date [1]. Indeed, the quasi-realistic models utilizing this formalism, constructed nearly two decades ago [2-4], have been utilised to explore how many of the issues pertaining to the phenomenology of the Standard Model and Grand Unified Theories may arise from string theory, including: fermion masses, mixing and $\mathrm{CP}$-violation; neutrino masses; proton stability; gauge coupling unification; supersymmetry breaking and smatter degeneracy [1]. Additionally, this construction gives rise to models in which the Standard Model charged spectrum below the string scale consist solely of that of the Minimal Supersymmetric Standard Model [3]. The phenomenological free fermionic string models therefore serve as a laboratory in which we can investigate how the Standard Model data may be obtained from a fundamental theory. In turn, these models are used to reveal general properties of string theory. In this vein, duality under the exchange of spinors and vectors of the $S O(10)$ GUT group was discovered [5]. This, and other, duality symmetries in the string vacua space, indicate that the string models live in a connected space. Thus, while from point of view of the effective low energy field theory limit of the string vacua, the spinor-vector dual pairs correspond to nonequivalent theories, the vacua are connected in string theory. Essentially, we can say that in string theory the duality map interchanges between massless and massive string states, hence inducing the duality map between vacua that in the effective low energy field theory correspond to completely different phenomenology.

The need to understand better the properties of the free fermionic models is evident. An important feature of the realistic free fermionic models is their underlying $Z_{2} \times Z_{2}$ orbifold structure [6,7]. Furthermore, a vital distinction of the quasi-realistic free fermionic models is that the initial gauge symmetry at the level of the toroidal compactification with four dimensional $N=4$ space-time supersymmetry, which is then moded by the $Z_{2} \times Z_{2}$ orbidold to $N=1$, correspond to $S O(16) \times S O(16)$, rather than the more conventional $E_{8} \times E_{8}$ symmetry, which is usually the initial symmetry in orbifold constructions. This distinction has important phenomenological consequence that have been elaborated elsewhere [8]. In the free fermionic formalism the two cases can be seen to arise from a discreet choice of a Generalised GSO (GGSO) projection coefficient. Alternatively, as has been demonstrated in [7] by studying the respective partition functions, the two cases can be connected by a $Z_{2} \times Z_{2}$ orbifold. In the ten dimensional case the relevant GGSO phase correspond to the discreet choice between the $N=1$ supersymmetric heterotic $E_{8} \times E_{8}$ string [9] versus the non-supersymmetric $S O(16) \times S O(16)$ heterotic-string vacuum [10].

In the free fermionic formalism the relevant phase is the GGSO phase, which is responsible for the projection of the spinorial representations in the decomposition of the adjoint of $E_{8} \times E_{8}$ under $S O(16) \times S O(16)[6,7]$, hence inducing the reduction of the symmetry from $E_{8} \times E_{8}$ to $S O(16) \times S O(16)$. The discreet GGSO choice is 
responsible for this projection in the ten and four dimensional free fermion models. Alternatively, one can formulate the same projection at the free fermion point as orbifolding by a fermion number that acts on the gauge degrees of freedom coupled with a fermion number acting on the internal lattice, in the four dimensional case [7], or on the space-time degrees of freedom in the ten dimensional case [10]. The natural question is what is the relation between the cases. In this paper we address this question by writing explicitly the partition function of these vacua at the free fermion point, and subsequently at the general point of moduli space. This may assist on better understanding of the internal spaces of these vacua and possibly the geometrical structure that they give rise to.

We first formulate the orbifold projection from the $E_{8} \times E_{8}$ to $S O(16) \times S O(16)$ gauge symmetry as a fermion number acting on the gauge degrees of freedom coupled with a shift in the internal the internal lattice. We then construct the partition functions corresponding to the model with the internal shift and demonstrate how it can be interpolated with the corresponding nonsupersymmetric vacuum. The results presented here therefore elucidate the role of the free phase in the free fermionic models and how its operation is translated to the orbifold construction. One can then hope to be able to retrieve some of the successful phenomenological and duality features of the free fermion models in the orbifold models. In the reverse direction, one may hope to gain some insight into the geometrical structures that underlie the free fermion models, in particular in relation to the dualities and vacuum selection.

Our paper is organised as follows. In section 2 we review the structure of the quasi-realistic free fermionic models, and in particular the characteristics that are relevant for the analysis here. In section 3 we discuss the partition functions underlying the NAHE-based free fermionic models. In section 4 we discuss the interpolations among the NAHE-based partition functions. Section 5 concludes the paper.

\section{Review of free fermionic models}

In this section we discuss some of the relevant features of the free fermionic models (see [1] for a more detailed introduction). In the free fermionic formulation of the heterotic string in four dimensions all the world-sheet degrees of freedom required to cancel the conformal anomaly are represented in terms of free fermions propagating on the string world-sheet [11]. In the light-cone gauge the world-sheet field content consists of two transverse left- and right-moving space-time coordinate bosons, $X_{1,2}^{\mu}$ and $\bar{X}_{1,2}^{\mu}$, and their left-moving fermionic superpartners $\psi_{1,2}^{\mu}$, and additional 62 purely internal Majorana-Weyl fermions, of which 18 are left-moving,

$$
\chi^{1, . ., 6}, \quad y^{1, \ldots, 6}, \quad \omega^{1, \ldots, 6}
$$

and 44 are right-moving

$$
\bar{y}^{1, \ldots, 6}, \quad \bar{\omega}^{1, \ldots, 6}, \quad \bar{\psi}^{1, . ., 5}, \quad \bar{\eta}^{1,2,3}, \quad \bar{\phi}^{1, \ldots, 8} .
$$


Under parallel transport around a non-contractible loop on the toroidal world-sheet the fermionic fields pick up a phase, $f \rightarrow-\mathrm{e}^{i \pi \alpha(f)} f, \quad \alpha(f) \in(-1,+1]$. Each set of specified phases for all world-sheet fermions, around all the non-contractible loops is called the spin structure of the model. Such spin structures are usually given in the form of 64 dimensional boundary condition vectors, with each element of the vector specifying the phase of the corresponding world-sheet fermion. The basis vectors are constrained by string consistency requirements and completely determine the vacuum structure of the model. The physical spectrum is obtained by applying the generalised GSO projections. The boundary condition basis defining a typical "realistic free fermionic heterotic string model" is constructed in two stages. The first stage consists of the NAHE set, which is a set of five boundary condition basis vectors, $\left\{1, S, b_{1}, b_{2}, b_{3}\right\}[12]$

$$
\begin{gathered}
S=\left\{\psi^{1,2}, \chi^{1, \ldots, 6}\right\}, \quad b_{1}=\left\{\psi^{1,2}, \chi^{1,2}, y^{3, . .6} \mid \bar{y}^{3, . .6}, \bar{\psi}^{1, . .5,}, \bar{\eta}^{1}\right\} \\
b_{2}=\left\{\psi^{1,2}, \chi^{3,4}, y^{1,2}, \omega^{5,6} \mid \bar{y}^{1,2}, \bar{\omega}^{5,6}, \bar{\psi}^{1, . .5}, \bar{\eta}^{2}\right\} \\
b_{3}=\left\{\psi^{1,2}, \chi^{3,4}, \omega^{1, . .4} \mid \bar{\omega}^{1, . .4}, \bar{\psi}^{1, . .5,}, \bar{\eta}^{3}\right\}
\end{gathered}
$$

where fields with $\alpha(f)=1$ are indicated explicitly The gauge group after imposing the GSO projections induced by the NAHE set is $\mathrm{SO}(10) \times \mathrm{SO}(6)^{3} \times \mathrm{E}_{8}$ with $N=1$ supersymmetry.

The second stage of the construction consists of adding to the NAHE set three (or four) additional boundary condition basis vectors, typically denoted by $\{\alpha, \beta, \gamma\}$. These additional basis vectors reduce the number of generations to three chiral generations, one from each of the sectors $b_{1}, b_{2}$ and $b_{3}$, and simultaneously break the four dimensional gauge group. The assignment of boundary conditions to $\left\{\bar{\psi}^{1, \cdots, 5}\right\}$ breaks $\mathrm{SO}(10)$ to one of its subgroups $\mathrm{SU}(5) \times \mathrm{U}(1)$ [2], $\mathrm{SO}(6) \times \mathrm{SO}(4)$ [4], $\mathrm{SU}(3) \times \mathrm{SU}(2) \times \mathrm{U}(1)^{2}[3]$ or $\mathrm{SU}(3) \times \mathrm{SU}(2)^{2} \times \mathrm{U}(1)[13]$.

The correspondence of the NAHE-based free fermionic models with the orbifold construction is illustrated by extending the NAHE set, $\left\{1, S, b_{1}, b_{2}, b_{3}\right\}$, by one additional boundary condition basis vector [6],

$$
\xi_{1}=\left\{\bar{\psi}^{1, . .5}, \bar{\eta}^{1,2,3}\right\},
$$

To construct the model in the orbifold formulation one starts with the compactification on a torus with nontrivial background fields [14]. The subset of basis vectors

$$
\left\{1, S, \xi_{1}, \xi_{2}\right\}, \quad \xi_{2}=1+b_{1}+b_{2}+b_{3}
$$

generates a toroidally-compactified model with $N=4$ space-time supersymmetry and $\mathrm{SO}(12) \times \mathrm{E}_{8} \times \mathrm{E}_{8}$ gauge group. Adding the two basis vectors $b_{1}$ and $b_{2}$ to the set (2.2) corresponds to the $Z_{2} \times Z_{2}$ orbifold model with standard embedding. Starting from the Narain model with $\mathrm{SO}(12) \times \mathrm{E}_{8} \times \mathrm{E}_{8}$ symmetry [14], and applying the $Z_{2} \times Z_{2}$ 
twist on the internal coordinates, reproduces the spectrum of the free-fermion model with the six-dimensional basis set $\left\{1, S, \xi_{1}, \xi_{2}, b_{1}, b_{2}\right\}$. The Euler characteristic of this model is 48 with $h_{11}=27$ and $h_{21}=3$.

The effect of the additional basis vector $\xi_{1}$ of eq. (2.1), is to separate the gauge degrees of freedom, spanned by the world-sheet fermions $\left\{\bar{\psi}^{1, \cdots, 5}, \bar{\eta}^{1}, \bar{\eta}^{2}, \bar{\eta}^{3}, \bar{\phi}^{1, \cdots, 8}\right\}$, from the internal compactified degrees of freedom $\{y, \omega \mid \bar{y}, \bar{\omega}\}^{1, \cdots, 6}$. In the "realistic free fermionic models" this is achieved by the vector $2 \gamma[6]$

$$
2 \gamma=\left\{\bar{\psi}^{1, \ldots, 5}, \bar{\eta}^{1,2,3}, \bar{\phi}^{1 \ldots, 4}\right\}
$$

This vector breaks $E_{8} \times E_{8}$ gauge symmetry down to $S O(16) \times S O(16)$, whereas $Z_{2} \times Z_{2}$ orbifold further breaks it down to $S O(4)^{3} \times S O(10) \times U(1)^{3} \times S O(16)$. The orbifold still yields a model with 24 generations, eight from each twisted sector, but now the generations are in the chiral 16 representation of $\mathrm{SO}(10)$, rather than in the 27 of $\mathrm{E}_{6}$. The same model can be realized with the set $\left\{1, S, \xi_{1}, \xi_{2}, b_{1}, b_{2}\right\}$, by projecting out the $16 \oplus \overline{16}$ from the $\xi_{1}$-sector taking

$$
c\left(\begin{array}{l}
\xi_{1} \\
\xi_{2}
\end{array}\right) \rightarrow-c\left(\begin{array}{l}
\xi_{1} \\
\xi_{2}
\end{array}\right),
$$

where $c\left(\begin{array}{l}\xi_{1} \\ \xi_{2}\end{array}\right)$ is a GGSO phase appearing in the partition function. This choice also projects out the massless vector bosons in the 128 of $\mathrm{SO}(16)$ in the hidden-sector $\mathrm{E}_{8}$ gauge group, thereby breaking the $\mathrm{E}_{6} \times \mathrm{E}_{8}$ symmetry to $\mathrm{SO}(10) \times \mathrm{U}(1) \times \mathrm{SO}(16)$.

The freedom in (2.4) corresponds to a discrete torsion in the free fermionic model. At the level of the $N=4$ Narain model generated by the set (2.2), we can define two models, $Z_{+}$and $Z_{-}$, depending on the sign of the discrete torsion in eq. (2.4). The first, say $Z_{+}$, produces the $\mathrm{E}_{8} \times \mathrm{E}_{8}$ model, whereas the second, say $Z_{-}$, produces the $\mathrm{SO}(16) \times \mathrm{SO}(16)$ model. However, the $Z_{2} \times Z_{2}$ twist acts identically in the two models, and their physical characteristics differ only due to the discrete torsion eq. (2.4).

The projection induced by eqs. (2.3), or (2.4), has important phenomenological consequences in the free fermionic constructions that are relevant for orbfiold models. In the case of $Z_{+}$the action of the $Z_{2} \times Z_{2}$ orbifold is to break the observable $E_{8}$ symmetry to $E_{6} \times U(1)^{2}$. The chiral matter states are contained in the 27 representation of $E_{6}$, which decomposes as

$$
27=16_{\frac{1}{2}}+10_{-1}+1_{2}
$$

under the $S O(10) \times U(1)$ subgroup of $E_{6}$, where the spinorial 16 and vectorial 10 representations of $S O(10)$ contain the Standard Model fermion and Higgs states, respectively. The projection induced by (2.4) in $Z_{-}$entails that either the spinorial, or the vectorial, representation survives the GSO projection at a given fixed point. Hence, this projection operates a Higgs-matter splitting mechanism [8] in the phenomenological free fermionic models. 
In contra-distinction most of the heterotic orbifold models constructed to date are based on on the $E_{8} \times E_{8}$ heterotic-string compactified to four dimensions, and the breaking of the $E_{8}$ symmetry is induced by Wilson lines [15] (for constructions based on the $S O(32)$ heterotic-string see e.g. [16]). The $Z_{-}$choice in (2.4) also results in the breaking of the right-moving $N=2$ world-sheet symmetry, and is relevant in the spinor-vector duality, observed in ref. [5] in the framework of the free fermionic models. Thus, it would be beneficial to learn how to implement this projection in the orbifold models, which will open the way to construct new classes of quasi-realistic orbifold models, as well as to obtain a realisation of the spinor-vector duality in such models.

\section{Partition functions of NAHE-based models}

The partition functions corresponding to the $Z_{-}^{4 d}$ and $Z_{+}^{4 d}$ vacua after the compactification of the ten dimensional $E_{8} \times E_{8}$ heterotic superstring on the $S O(12)$ lattice at the special point of the moduli space are given by

$$
\begin{aligned}
Z_{-}^{4 d}=\frac{\left(V_{8}-S_{8}\right)}{\tau_{2}(\eta \bar{\eta})^{8}} \times & {\left[\left(\left|O_{12}\right|^{2}+\left|V_{12}\right|^{2}\right)\left(\bar{O}_{16} \bar{O}_{16}+\bar{C}_{16} \bar{C}_{16}\right)\right.} \\
& +\left(\left|S_{12}\right|^{2}+\left|C_{12}\right|^{2}\right)\left(\bar{S}_{16} \bar{S}_{16}+\bar{V}_{16} \bar{V}_{16}\right) \\
& +\left(O_{12} \bar{V}_{12}+V_{12} \bar{O}_{12}\right)\left(\bar{S}_{16} \bar{V}_{16}+\bar{V}_{16} \bar{S}_{16}\right) \\
& \left.+\left(S_{12} \bar{C}_{12}+C_{12} \bar{S}_{12}\right)\left(\bar{O}_{16} \bar{C}_{16}+\bar{C}_{16} \bar{O}_{16}\right)\right],
\end{aligned}
$$

and

$$
Z_{+}^{4 d}=\frac{\left(V_{8}-S_{8}\right)}{\tau_{2}(\eta \bar{\eta})^{8}}\left[\left|O_{12}\right|^{2}+\left|V_{12}\right|^{2}+\left|S_{12}\right|^{2}+\left|C_{12}\right|^{2}\right]\left(\bar{O}_{16}+\bar{S}_{16}\right)\left(\bar{O}_{16}+\bar{S}_{16}\right),
$$

depending on the sign of the discrete torsion. Here we have written $Z_{ \pm}$in terms of level-one $\mathrm{SO}(2 n)$ characters (see, for instance [17])

$$
\begin{aligned}
O_{2 n} & =\frac{1}{2}\left(\frac{\vartheta_{3}^{n}}{\eta^{n}}+\frac{\vartheta_{4}^{n}}{\eta^{n}}\right), \\
V_{2 n} & =\frac{1}{2}\left(\frac{\vartheta_{3}^{n}}{\eta^{n}}-\frac{\vartheta_{4}^{n}}{\eta^{n}}\right), \\
S_{2 n} & =\frac{1}{2}\left(\frac{\vartheta_{2}^{n}}{\eta^{n}}+i^{-n} \frac{\vartheta_{1}^{n}}{\eta^{n}}\right), \\
C_{2 n} & =\frac{1}{2}\left(\frac{\vartheta_{2}^{n}}{\eta^{n}}-i^{-n} \frac{\vartheta_{1}^{n}}{\eta^{n}}\right)
\end{aligned}
$$

and the expressions of the form $\left|O_{12}\right|^{2}$ mean $O_{12} \bar{O}_{12}$. These two models can actually be connected by the orbifold [7]

$$
Z_{-}=Z_{+} / a \otimes b
$$


with

$$
\begin{aligned}
a & =(-1)^{F_{\mathrm{L}}^{\mathrm{int}}+F_{\xi}^{1}} \\
b & =(-1)^{F_{\mathrm{L}}^{\mathrm{int}}+F_{\xi}^{2}}
\end{aligned}
$$

Here $F_{\mathrm{L}}$ is the fermion number for the "left" component in the expression of the internal lattice i.e., the only nontrivial action of this operator is $F_{\mathrm{L}} S_{12}=S_{12}$ and $F_{\mathrm{L}} C_{12}=C_{12}$. The operators $F_{\xi}^{1}$ and $F_{\xi}^{2}$ are fermion number operators in the first and the second gauge factors respectively. The orbifold projection given in eqs (3.4) and (3.5) is defined at the free fermionic point in the moduli space since $Z_{+}^{4 d}$ and $Z_{-}^{4 d}$ are expressed at this point. However, it can be generalised to an arbitrary point in the moduli space and hence can be employed to construct orbifold models that originate from the $Z_{-}^{4 d}$ partition function, in analogy to the case in the free fermionic constructions.

The analogous partition function $Z_{+}^{9 d}$ in the case of one compactified dimension at a general point of moduli space is given by

$$
Z_{+}^{9 d}=\frac{\left(V_{8}-S_{8}\right)}{\tau_{2}^{\frac{7}{2}}(\eta \bar{\eta})^{8}} \Lambda_{m, n}\left(\bar{O}_{16}+\bar{S}_{16}\right)\left(\bar{O}_{16}+\bar{S}_{16}\right)
$$

where

$$
\Lambda_{m, n}=q^{\frac{\alpha^{\prime}}{4} p_{L}^{2}} \bar{q}^{\frac{\alpha^{\prime}}{4} p_{\mathrm{R}}^{2}}, \quad p_{\mathrm{L}, \mathrm{R}}=\frac{m}{R} \pm \frac{n R}{\alpha^{\prime}}
$$

applying the orbifold projections

$$
\begin{aligned}
& Z_{2}: g=(-1)^{F_{\xi^{1}}} \delta_{1}, \\
& Z_{2}^{\prime}: g^{\prime}=(-1)^{F^{2}} \delta_{1} .
\end{aligned}
$$

where

$$
\delta_{1}: X^{9} \rightarrow X^{9}+\pi R^{9}, \quad \Rightarrow \quad \Lambda_{m n} \rightarrow(-1)^{m} \Lambda_{m n} .
$$

in $Z_{+}^{9 d}$ produces the $Z_{-}^{9 d}$ partition function given by

$$
\begin{aligned}
& Z_{-}^{9 d}=\frac{\left(V_{8}-S_{8}\right)}{\tau_{2}^{\frac{7}{2}}(\eta \bar{\eta})^{8}}\left[\quad \Lambda_{2 m, n} \quad\left(\bar{O}_{16} \bar{O}_{16}+\bar{C}_{16} \bar{C}_{16}\right)\right. \\
& +\Lambda_{2 m+1, n} \quad\left(\bar{S}_{16} \bar{S}_{16}+\bar{V}_{16} \bar{V}_{16}\right) \\
& +\Lambda_{2 m, n+\frac{1}{2}} \quad\left(\bar{S}_{16} \bar{V}_{16}+\bar{V}_{16} \bar{S}_{16}\right) \\
& \left.+\Lambda_{2 m+1, n+\frac{1}{2}}\left(\bar{O}_{16} \bar{C}_{16}+\bar{C}_{16} \bar{O}_{16}\right)\right] \text {. }
\end{aligned}
$$

Let us note that the internal fermion number operator which appears in (3.5) is now replaced by the shift along the compact dimension, given in (3.9). 
We note here that the shift given by delta differs from the shifts that were found in ref. [7] to reproduce the partition function of the $S O(12)$ lattice at the maximally symmetric free fermionic point, given in eq.(3.2). Since the string contains momentum and winding modes along a compactified coordinate, one can shift the coordinate along either, and also allow shifts that mix the momentum and winding modes [18]. Indeed, the precise identification of the lattice at the free fermionic point is obtained for shifts that mix momentum and winding modes. Here we restrict ourselves to the simpler shifts and incorporation of other shifts is left for a future work.

Let us consider partition functions (3.6) and (3.10) at the special (free fermionic) point of the moduli space i.e.,

$$
Z_{+}^{9 d}=\frac{\left(V_{8}-S_{8}\right)}{\tau_{2}^{\frac{7}{2}}(\eta \bar{\eta})^{8}}\left(\left|O_{2}\right|^{2}+\left|V_{2}\right|^{2}+\left|S_{2}\right|^{2}+\left|C_{2}\right|^{2}\right)\left(\bar{O}_{16}+\bar{S}_{16}\right)\left(\bar{O}_{16}+\bar{S}_{16}\right)
$$

and

$$
\begin{aligned}
Z_{-}^{9 d}=\frac{\left(V_{8}-S_{8}\right)}{\tau_{2} \frac{7}{2}(\eta \bar{\eta})^{8}} \times & {\left[\left(\left|O_{2}\right|^{2}+\left|V_{2}\right|^{2}\right)\left(\bar{O}_{16} \bar{O}_{16}+\bar{C}_{16} \bar{C}_{16}\right)\right.} \\
& +\left(\left|S_{2}\right|^{2}+\left|C_{2}\right|^{2}\right)\left(\bar{S}_{16} \bar{S}_{16}+\bar{V}_{16} \bar{V}_{16}\right) \\
& +\left(O_{2} \bar{V}_{2}+V_{2} \bar{O}_{2}\right)\left(\bar{S}_{16} \bar{V}_{16}+\bar{V}_{16} \bar{S}_{16}\right) \\
& \left.+\left(S_{2} \bar{C}_{2}+C_{2} \bar{S}_{2}\right)\left(\bar{O}_{16} \bar{C}_{16}+\bar{C}_{16} \bar{O}_{16}\right)\right]
\end{aligned}
$$

In this case the $Z_{2} \times Z_{2}^{\prime}$ orbifold operation is again given by (3.5) where $F_{L}^{\text {int }}$ acts on $S_{2}$ and $C_{2}$ of the internal one - dimensional lattice. Equations (3.11) and (3.12) therefore have the same structure as the corresponding four dimensional equations (3.1) and (3.2) respectively.

We now comment on the ten dimensional case. In terms of the free fermionic formalism both $Z_{+}$and $Z_{-}$models are generated by the set of basis vectors $\left\{1, \xi_{1}, \xi_{2}\right\}$. The $S$-sector is obtained as a combination of the basis vectors with $S=1+\xi_{1}+\xi_{2}$. As a result the GGSO coefficient $c\left(\begin{array}{l}S \\ \xi_{1}\end{array}\right)$ is proportional to $c\left(\begin{array}{l}\xi_{2} \\ \xi_{1}\end{array}\right)$. Consequently, the choice of the GGSO coefficient

$$
c\left(\begin{array}{l}
\xi_{2} \\
\xi_{1}
\end{array}\right)=-1
$$

reduces the $E_{8} \times E_{8}$ gauge symmetry to $S O(16) \times S O(16)$ as well as projects out the space-time supersymmetry generator from the $S$-sector. In terms of $c\left(\begin{array}{l}\xi_{1} \\ \xi_{2}\end{array}\right)$ the free fermionic partition function takes the form

$$
Z^{10 d}=\frac{1}{\tau_{2}^{4}(\eta \bar{\eta})^{8}} \frac{1}{2^{3}}\left\{\left(\theta_{3}^{4}-\theta_{4}^{4}-\theta_{2}^{4}-\theta_{1}^{4}\right)\left(\bar{\theta}_{3}^{16}+\bar{\theta}_{4}^{16}+\bar{\theta}_{2}^{16}+\bar{\theta}_{1}^{16}\right)\right.
$$




$$
\begin{aligned}
& +\left[\theta_{3}^{4}-\theta_{4}^{4}-c\left(\begin{array}{l}
\xi_{1} \\
\xi_{2}
\end{array}\right)\left(\theta_{2}^{4}+\theta_{1}^{4}\right)\right]\left[\bar{\theta}_{3}^{8} \bar{\theta}_{4}^{8}+\bar{\theta}_{4}^{8} \bar{\theta}_{3}^{8}+c\left(\begin{array}{l}
\xi_{1} \\
\xi_{2}
\end{array}\right)\left(\bar{\theta}_{2}^{8} \bar{\theta}_{1}^{8}+\bar{\theta}_{1}^{8} \bar{\theta}_{2}^{8}\right)\right] \\
& +\left[\theta_{3}^{4}-c\left(\begin{array}{l}
\xi_{1} \\
\xi_{2}
\end{array}\right)\left(\theta_{4}^{4}+\theta_{1}^{4}\right)-\theta_{2}^{4}\right]\left[\bar{\theta}_{2}^{8} \bar{\theta}_{3}^{8}+\bar{\theta}_{3}^{8} \bar{\theta}_{2}^{8}+c\left(\begin{array}{l}
\xi_{1} \\
\xi_{2}
\end{array}\right)\left(\bar{\theta}_{4}^{8} \bar{\theta}_{1}^{8}+\bar{\theta}_{1}^{8} \bar{\theta}_{4}^{8}\right)\right] \\
& \left.\left[c\left(\begin{array}{l}
\xi_{1} \\
\xi_{2}
\end{array}\right)\left(\theta_{3}^{4}-\theta_{1}^{4}\right)-\theta_{4}^{4}-\theta_{2}^{4}\right]\left[\bar{\theta}_{2}^{8} \bar{\theta}_{4}^{8}+\bar{\theta}_{4}^{8} \bar{\theta}_{2}^{8}+c\left(\begin{array}{l}
\xi_{1} \\
\xi_{2}
\end{array}\right)\left(\bar{\theta}_{3}^{8} \bar{\theta}_{1}^{8}+\bar{\theta}_{1}^{8} \bar{\theta}_{3}^{8}\right)\right]\right\}
\end{aligned}
$$

The choice $c\left(\begin{array}{l}\xi_{1} \\ \xi_{2}\end{array}\right)=1$ of the GGSO coefficient corresponds to the partition function $Z_{+}$and the choice $c\left(\begin{array}{c}\xi_{1} \\ \xi_{2}\end{array}\right)=-1$ corresponds to $Z_{-}$. In terms of the characters of eq. (3.3) $Z_{+}$takes the form

$$
Z_{+}^{10 d}=\frac{\left(V_{8}-S_{8}\right)}{\tau_{2}^{4}(\eta \bar{\eta})^{8}}\left(\bar{O}_{16}+\bar{S}_{16}\right)\left(\bar{O}_{16}+\bar{S}_{16}\right)
$$

and $Z_{-}^{10 d}$

$$
\begin{aligned}
\left.Z_{-}^{10 d}=\frac{1}{\tau_{2}^{4}(\eta \bar{\eta})^{8}}\right] & {\left[V_{8}\left(\bar{O}_{16} \bar{O}_{16}+\bar{C}_{16} \bar{C}_{16}\right)\right.} \\
- & S_{8}\left(\bar{S}_{16} \bar{S}_{16}+\bar{V}_{16} \bar{V}_{16}\right) \\
+ & O_{8}\left(\bar{S}_{16} \bar{V}_{16}+\bar{V}_{16} \bar{S}_{16}\right) \\
- & \left.C_{8}\left(\bar{O}_{16} \bar{C}_{16}+\bar{C}_{16} \bar{O}_{16}\right)\right] .
\end{aligned}
$$

The projection to be implemented in $Z_{+}$to obtain $Z_{-}$is

$$
\frac{1+(-1)^{F+F_{\xi_{1}}}}{2} \times \frac{1+(-1)^{F+F_{\xi_{2}}}}{2},
$$

with $F$ being the space-time fermion number and $F_{\xi_{1,2}}$ as before. Therefore the projection in the ten dimensional case reproduces the partition function of the nonsupersymmetric $S O(16) \times S O(16)$ heterotic string.

In order to see what happens for the case of compactification to nine dimensions one can rewrite the partition functions (3.11) and (3.12) in the form similar to (3.13) i.e., therms of $\theta$ functions. The one can show that the difference between these partition functions is due to a choice of the discrete torsion $c\left(\begin{array}{l}\xi_{1} \\ \xi_{2}\end{array}\right)= \pm 1$. One can conclude that in the free fermionic construction the same phase that reduces the gauge symmetry in the compactified model from $E_{8} \times E_{8}$ to $S O(16) \times S O(16)$, uniquely in the uncompactified case also projects out the space-time supersymmetry generator. The natural question is therefore what is the relation in the compactified case between the supersymmetric an non-supersymmetric models. We turn to address this question in the next section. 


\section{Interpolations}

To explore the connection between the two models we explore the following compactifications. First we study the compactification of the heterotic $E_{8} \times E_{8}$ on a circle $S_{1}$ moded by $Z_{2} \times Z_{2}^{\prime}$ orbifold as in (3.9). The resulting theory is a heterotic-string with $N=1$ supersymmetry and $S O(16) \times S O(16)$ gauge symmetry. Schematically, we have

$$
\frac{\left(\text { Het } E_{8} \times E_{8} \text { on } S_{1}\right)}{Z_{2} \times Z_{2}^{\prime}}=\text { het } S O(16) \times S O(16) \text { on } S_{1} \text { with } N=1 \quad \text { SUSY }
$$

Next, we compactify this heterotic $S O(16) \times S O(16)$ model on another circle $S_{1}^{\prime}$ moded by $Z_{2}^{\prime}$ with the shift

$$
Z_{2}^{\prime \prime}: g^{\prime \prime}=(-1)^{F+F_{\xi_{1}}+F_{\xi_{2}}} \delta_{2}
$$

and

$$
\delta_{2}: X_{8} \rightarrow X_{8}+\pi R_{8} \Rightarrow \Gamma_{m n} \rightarrow(-1)^{m} \Gamma_{m n} .
$$

The fermion numbers $F_{\xi_{1}}$ and $F_{\xi_{2}}$ in eq. (4.2) are as before, whereas the fermion number $F$ refers to the space-time fermion number. We then demonstrate that the limit $R_{8} \rightarrow 0$ of the $S O(16) \times S O(16)$ non-supersymmetric heterotic string in eight dimensions produces the partition function of the ten dimensional $S O(16) \times$ $S O(16)$ non-supersymmetric heterotic string compactified on a circle $S_{1}$, moded by the $Z_{2} \times Z_{2}^{\prime}$ orbifold. Alternatively, the decompactification limit $R_{8} \rightarrow \infty$ of the nonsupersymmetric heterotic string compactified to eight dimensions, interpolates to the a supersymmetric $S O(16) \times S O(16)$ heterotic-string in nine dimensions, moded by the $Z_{2} \times Z_{2}^{\prime}$ orbifold.

To demonstrate these interpolations in a manner similar to $[19,20]$ we start with the partition function of the $E_{8} \times E_{8}$ heterotic-string compactified on one $S_{1}$. The partition function of this vacuum is given in eq. (3.11). We then implement the $Z_{2} \times Z_{2}^{\prime}$ orbifold projection given in eq. (3.5). Operating with the $Z_{2} \times Z_{2}^{\prime}$ introduces a discrete torsion, $\epsilon= \pm 1$, in the partition function, which is an undefined sign between independent modular orbits. The choice of the negative sign produces a supersymmetric nine dimensional string vacuum with $S O(32)$ gauge group, with

$$
\bar{Z}_{-}^{9 d}=\frac{\left(V_{8}-S_{8}\right)}{\tau_{2}^{\frac{7}{2}}(\eta \bar{\eta})^{8}}\left[\left(\bar{O}_{32} P_{+}+\bar{S}_{32} P_{-}\right) \Lambda+\left(\bar{V}_{32} P_{-}+\bar{C}_{32} P_{+}\right) \Lambda_{\frac{1}{2}}\right]
$$

where we introduced the notation

$$
P_{ \pm}=\frac{1}{2}\left(1 \pm(-1)^{m}\right)
$$

and

$$
\begin{aligned}
\Lambda & \equiv \Lambda_{m, n} \\
\Lambda_{\frac{1}{2}} & \equiv \Lambda_{m, n+\frac{1}{2}}
\end{aligned}
$$


We note that the \pm index here refers to the choice of the discrete torsion, $\epsilon= \pm 1$, and differs from the \pm index used previously which referred to the choice of the sign in eq. (2.4). The choice $\epsilon=+1$ yields

$$
\begin{gathered}
\bar{Z}_{+}^{9 d=} \frac{\left(V_{8}-S_{8}\right)}{\tau_{2}^{\frac{7}{2}}(\eta \bar{\eta})^{8}}\left\{\left[\left(\bar{O}_{16} \bar{O}_{16}+\bar{C}_{16} \bar{C}_{16}\right) P_{+}+\left(\bar{S}_{16} \bar{S}_{16}+\bar{V}_{16} \bar{V}_{16}\right) P_{-}\right] \Lambda+\right. \\
\left.\left[\left(\bar{C}_{16} \bar{O}_{16}+\bar{O}_{16} \bar{C}_{16}\right) P_{+}+\left(\bar{V}_{16} \bar{S}_{16}+\bar{S}_{16} \bar{V}_{16}\right) P_{-}\right] \Lambda_{\frac{1}{2}}\right\}
\end{gathered}
$$

which is a generalization of (3.12) to an arbitrary point in the moduli space. The partition function in eq. (4.7) is our starting point for interpolating between the relevant string vacua discussed above. We next mod out the partition function given in eq. (4.7) by the $Z_{2}^{\prime \prime}$ orbifold given in eq. (4.2). The resulting partition function is given by

$$
\begin{aligned}
& \bar{Z}_{+}^{8 d}=\frac{1}{\tau_{2}^{3}(\eta \bar{\eta})^{8}}\left[\left\{\quad V_{8} \quad\left[\left(\bar{O}_{16} \bar{O}_{16}+\bar{C}_{16} \bar{C}_{16}\right) P_{+}+\left(\bar{S}_{16} \bar{S}_{16}+\bar{V}_{16} \bar{V}_{16}\right) P_{-}\right] \Lambda\right.\right. \\
& \left.-S_{8} \quad\left[\left(\bar{C}_{16} \bar{O}_{16}+\bar{O}_{16} \bar{C}_{16}\right) P_{+}+\left(\bar{V}_{16} \bar{S}_{16}+\bar{S}_{16} \bar{V}_{16}\right) P_{-}\right] \Lambda_{\frac{1}{2}}\right\} P_{+} \Gamma \\
& +\left\{V_{8}\left[\left(\bar{O}_{16} \bar{C}_{16}+\bar{C}_{16} \bar{O}_{16}\right) P_{+}+\left(\bar{V}_{16} \bar{S}_{16}+\bar{S}_{16} \bar{V}_{16}\right) P_{-}\right] \Lambda_{\frac{1}{2}}\right. \\
& \left.-S_{8}\left[\left(\bar{O}_{16} \bar{O}_{16}+\bar{C}_{16} \bar{C}_{16}\right) P_{+}+\left(\bar{S}_{16} \bar{S}_{16}+\bar{V}_{16} \bar{V}_{16}\right) P_{-}\right] \Lambda\right\} P_{-} \Gamma \\
& +\left\{O_{8}\left[\left(\bar{O}_{16} \bar{C}_{16}+\bar{C}_{16} \bar{O}_{16}\right) P_{-}+\left(\bar{V}_{16} \bar{S}_{16}+\bar{S}_{16} \bar{V}_{16}\right) P_{+}\right] \Lambda_{\frac{1}{2}}\right. \\
& \text { - } \left.C_{8} \quad\left[\left(\bar{O}_{16} \bar{O}_{16}+\bar{C}_{16} \bar{C}_{16}\right) P_{-}+\left(\bar{V}_{16} \bar{V}_{16}+\bar{S}_{16} \bar{S}_{16}\right) P_{+}\right] \Lambda\right\} P_{+} \Gamma_{\frac{1}{2}} \\
& +\left\{O_{8}\left[\left(\bar{O}_{16} \bar{O}_{16}+\bar{C}_{16} \bar{C}_{16}\right) P_{-}+\left(\bar{V}_{16} \bar{V}_{16}+\bar{S}_{16} \bar{S}_{16}\right) P_{+}\right] \Lambda\right. \\
& \text { - } \left.\left.C_{8}\left[\left(\bar{O}_{16} \bar{C}_{16}+\bar{C}_{16} \bar{O}_{16}\right) P_{-}+\left(\bar{V}_{16} \bar{S}_{16}+\bar{S}_{16} \bar{V}_{16}\right) P_{-}\right] \Lambda_{\frac{1}{2}}\right\} P_{+} \Gamma_{\frac{1}{2}}\right]
\end{aligned}
$$

We can now study the various limits as $R_{8} \rightarrow 0$ and $R_{8} \rightarrow \infty$, where $R_{8}$ is the radius of the $\Gamma$ lattice which accounts for the spontaneous supersymmetry breaking. These limits are:

\begin{tabular}{r|r}
$R_{8} \rightarrow \infty$ & $R_{8} \rightarrow 0$ \\
\hline \hline$\Gamma \rightarrow 1$ & $\Gamma \rightarrow 1$ \\
$(-1)^{m} \Gamma \rightarrow 0$ & $(-1)^{m} \Gamma \rightarrow 1$ \\
$\Gamma_{\frac{1}{2}} \rightarrow 0$ & $\Gamma_{\frac{1}{2}} \rightarrow 1$ \\
$(-1)^{m} \Gamma_{\frac{1}{2}} \rightarrow 0$ & $(-1)^{m} \Gamma_{\frac{1}{2}} \rightarrow 1$ \\
\hline
\end{tabular}

and $\frac{1}{\tau_{2}^{3}(\eta \bar{\eta})^{8}} \rightarrow \frac{1}{\tau_{2}^{\frac{7}{2}}(\eta \bar{\eta})^{8}}$

Therefore, in the limit $R_{8} \rightarrow \infty$ we recover the partition function in eq. (4.7), 
whereas the limit $R_{8} \rightarrow 0$ yields

$$
\begin{aligned}
\bar{Z}_{R_{8} \rightarrow 0}^{9 d}=\frac{1}{\tau_{2}^{\frac{7}{2}}(\eta \bar{\eta})^{8}}\left(\left\{\begin{array}{lll}
\{ & V_{8} & {\left[\left(\bar{O}_{16} \bar{O}_{16}+\bar{C}_{16} \bar{C}_{16}\right) P_{+}+\left(\bar{S}_{16} \bar{S}_{16}+\bar{V}_{16} \bar{V}_{16}\right) P_{-}\right] \Lambda(4.10)} \\
- & S_{8} & {\left[\left(\bar{C}_{16} \bar{O}_{16}+\bar{O}_{16} \bar{C}_{16}\right) P_{+}+\left(\bar{V}_{16} \bar{S}_{16}+\bar{S}_{16} \bar{V}_{16}\right) P_{-}\right] \Lambda_{\frac{1}{2}}}
\end{array}\right\}\right. \\
\left.+\left\{\begin{array}{ccc}
O_{8} & {\left[\left(\bar{O}_{16} \bar{C}_{16}+\bar{C}_{16} \bar{O}_{16}\right) P_{-}+\left(\bar{V}_{16} \bar{S}_{16}+\bar{S}_{16} \bar{V}_{16}\right) P_{+}\right] \Lambda_{\frac{1}{2}}} \\
- & C_{8} & {\left[\left(\bar{O}_{16} \bar{O}_{16}+\bar{C}_{16} \bar{C}_{16}\right) P_{-}+\left(\bar{V}_{16} \bar{V}_{16}+\bar{S}_{16} \bar{S}_{16}\right) P_{+}\right] \Lambda}
\end{array}\right\}\right)
\end{aligned}
$$

We need to understand now what theory this partition corresponds to and how it is connected to the non-supersymmetric non-tachyonic $S O(16) \times S O(16)$, or directly to the supersymmetric $E_{8} \times E_{8}$ heterotic-string theory. For this we consider the partition function of the ten dimensional $E_{8} \times E_{8}$ heterotic-string given in eq. (3.14) moded by the orbifold given in eq. (3.16). The resulting partition function is given in eq. (3.15) and is nonsupersymmetric $S O(16) \times S O(16)$ heterotic string. After that we compactify the non-supersymmetric $S O(16) \times S O(16)$ heterotic string on a circle $S_{1}$ and project with the freely acting $Z_{2} \times Z_{2}^{\prime}$ orbifold given in eq. (3.8). The partition function in this case, with the discrete torsion $\epsilon=-1$ exactly reproduces the one given in eq. (4.11), which is obtained from eq. (4.9) in the limit $R_{8} \rightarrow 0$.

Alternatively to the above derivation we can interpolate the vacua at the special point in the moduli space, with the lattice given in terms of the characters of eq. (3.3). Starting, say, from the $E_{8} \times E_{8}$ heterotic-string compactified to six dimensions, with

$$
Z_{+}^{6 d}=\frac{\left(V_{8}-S_{8}\right)}{\tau_{2}^{2}(\eta \bar{\eta})^{8}}\left[\left|O_{8}\right|^{2}+\left|V_{8}\right|^{2}+\left|S_{8}\right|^{2}+\left|C_{8}\right|^{2}\right]\left(\bar{O}_{16}+\bar{S}_{16}\right)\left(\bar{O}_{16}+\bar{S}_{16}\right)
$$

Further we perform a compactification on a circle $S_{1}$ with a $Z_{2} \times Z_{2}^{\prime}$ obifold action defined in (3.4), with $F_{L} S_{8}=S_{8}$ and $F_{L} C_{8}=C_{8}$ since now we are dealing with a four dimensional internal lattice. Further we compactify the model on another circle with $Z_{2}^{\prime \prime}$ orbifold action defined in (4.2). As it could be expected the result of this procedure is the same as in the case if the compactification to eight dimensions which was discussed above in this chapter with the following substitutions

$$
\begin{aligned}
P_{+} \Lambda & \rightarrow\left|O_{8}\right|^{2}+\left|V_{8}\right|^{2} \\
P_{-} \Lambda & \rightarrow\left|S_{8}\right|^{2}+\left|C_{8}\right|^{2} \\
P_{-} \Lambda_{\frac{1}{2}} & \rightarrow O_{8} \bar{V}_{8}+V_{8} \bar{O}_{8} \\
P_{+} \Lambda_{\frac{1}{2}} & \rightarrow S_{8} \bar{C}_{8}+C_{8} \bar{S}_{8} .
\end{aligned}
$$

To summarise, we note that the choice of the discrete phase in the free fermionic models is realised as an orbifold in the bosonic formalism. It is represented as a fermion number acting in the gauge sector, coupled with a shift in the internal lattice 
or, alternatively, with a fermion number acting in the internal lattice at the enhanced

symmetry point. The choice of the specific free phase in $c\left(\begin{array}{l}\xi_{1} \\ \xi_{2}\end{array}\right)= \pm 1$ determines many of the phenomenological properties of the free fermion models. Understanding how the projection induced by this phase is realised in the bosonic language will facilitate the reproduction of these features in orbifold models. In the ten dimensional free fermion model the choice $c\left(\begin{array}{l}\xi_{1} \\ \xi_{2}\end{array}\right)=-1$ projects out $N=1$ supersymmetry. On the other hand, in lower dimensional free fermion models with $c\left(\begin{array}{l}\xi_{1} \\ \xi_{2}\end{array}\right)=-1 N=1$ space-time supersymmetry is preserved, and the connection between the models is obscured. As we demonstrated here the bosonic representation of the models clarifies the situation and elucidated how the different cases are connected by interpolations. This demonstrates how the fermionic and bosonic representations of the string vacua provide complementary tools to reveal their properties.

\section{Conclusions}

String theory provides a unique framework to study how a spin 2 quantum field theory may be consistently incorporated with lower spin quantum field theories. As such it provides a unique arena to explore how the phenomenological properties of the Standard Model may arise from a theory of quantum gravity, and is hence the leading contender for a unified theory of gravity and the gauge interactions. Indeed string theory gives rise to a multitude of vacua that can in principle be relevant for low scale phenomenology. Among those the models constructed in the free fermionic formulations admit particularly appealing structure. The existence of a plethora of vacua would seem problematic from the low energy effective quantum field theory point of view. However, the lesson from the existence of symmetries under various duality transformations is that vacua that appear to be disconnected in the effective field theory limit may in fact be connected in the string theory due to the existence of the massive spectrum.

A key ingredient in the construction of the phenomenological free fermionic models is the GGSO phase, eq. (2.4). In the quasi-realistic four dimensional models this choice of phase accounts for the reduction of the GUT symmetry from $E_{6} \rightarrow S O(10)$, which results in Higgs-matter splitting [8]. Uniquely, in the ten dimensional case this choice of phase also projects out the space-time supersymmetry generator. While the free fermionic models possess phenomenologically attractive properties, it is of interest to obtain a representation of these models in a bosonic, i.e. orbifold, formulation. The reason being that the bosonic representation can reveal the geometrical structures underlying these models and hence to probe possible dynamical vacuum selection scenarios. Hence, it is of interest to learn how the breaking induced by the phase given in eq. (2.4) operates in the orbifold models. In general, we observe that this breaking is induced in the bosonic formalism by coupling the fermion number of the observable and hidden gauge degrees of freedom with a fermion number 
acting asymmetrically on the internal lattice at the self-dual point in the moduli space, or on the space-time fermions. Alternatively, the action in the internal dimensions can be represented as a mod two shift in the compactified coordinates. In the ten dimensional case the only possible internal action is on the space-time fermions, which breaks supersymmetry. We further addressed in this paper the question of how the models with the different actions of the fermion number in the internal spaces may be connected. This question is similar to the connection between the $E_{8} \times E_{8}$ and $S O(16) \times S O(16)$ heterotic strings, obtained by interpolations [19]. Indeed, we demonstrated in section 4 that the models produced by these differing actions can be connected by interpolations, i.e. that the relevant partition functions are reproduced in the appropriate limits. The phenomenologically appealing properties of the free fermionic models motivates their exploration in further depth. All the models studied to date in this context are supersymmetric and stable. However, it is plausible that unstable and nonsupersymmetric vacua can be instrumental for probing questions pertaining to dynamical vacuum selection scenarios. It is therefore vital to explore how the nonsupersymmetric models are incorporated in this picture, as well as in that of M-theory [21,22]. Understanding the bosonic equivalence of the free fermionic models, and their embedding in cosmological string solutions, offers the possibility of developing such a dynamical string vacuum selection scenario. We hope to return to these questions in the future.

\section{Acknowledgements}

We would like to thank Carlo Angelantonj for collaboration at the initial stage of the project and discussions throughout and Elisa Manno, Thomas Mohaupt and Cristina Timirgaziu for useful discussions. AEF thanks the Galileo Galilei Institute for hospitality. This work is supported in part by a STFC rolling grant $\mathrm{ST} / \mathrm{G} 00062 \mathrm{X} / 1$.

\section{References}

[1] For a recent review see e.g.: A.E. Faraggi, arXiv:0809.2641, and references therein.

[2] I. Antoniadis, J. Ellis, J. Hagelin and D.V. Nanopoulos Phys. Lett. B 231 (1989) 65.

[3] A.E. Faraggi, D.V. Nanopoulos and K. Yuan, Nucl. Phys. B 335 (1990) 347;

A.E. Faraggi, Phys. Rev. D 46 (1992) 3204;

A.E. Faraggi, Phys. Lett. B 278 (1992) 131; Phys. Lett. B 274 (1992) 47; Nucl. Phys. B 403 (1993) 101; Nucl. Phys. B 387 (1992) 239;

G.B. Cleaver, A.E. Faraggi and D.V. Nanopoulos, Phys. Lett. B 455 (1999) 
135;

G.B. Cleaver, A.E. Faraggi, D.V. Nanopoulos and J.W. Walker, Nucl. Phys. B 620 (2002) 259.

[4] I. Antoniadis, G.K Leontaris and J. Rizos, Phys. Lett. B 245 (1990) 161;

G.K Leontaris and J. Rizos, Nucl. Phys. B 554 (1999) 3;

B. Assel et al, arXiv:0910.3697.

[5] A.E.Faraggi, C. Kounnas and J. Rizos, Phys. Lett. B 648 (2007) 84; Nucl. Phys. B 774 (2007) 208; Nucl. Phys. B 799 (2008) 19;

T. Catelin-Jullien, A.E. Faraggi, C. Kounnas and J. Rizos, Nucl. Phys. B 812 (2009) 103 .

[6] A.E. Faraggi, Phys. Lett. B 326 (1994) 62;

P. Berglund et al, Phys. Lett. B 433 (1998) 269; Int. J. Mod. Phys. A 15 (2000) 1345 .

[7] A.E. Faraggi, Phys. Lett. B 544 (2002) 207;

A.E. Faraggi and E. Manno, arXiv:0908.2034. .

[8] A.E. Faraggi, Eur. Phys. Jour. C49 (2007) 803.

[9] D.J. Gross, J.A. Harvey, E.J. Martinec and R. Rohm, Nucl. Phys. B 256 (1985) 253.

[10] L.J. Dixon and J.A. Harvey, Nucl. Phys. B 274 (1986) 93;

L. Alvarez-Gaume, P.H. Ginsparg, G.W. Moore and C. Vafa, Phys. Lett. B 171 (1986) 155;

P.H. Ginsparg and C. Vafa, Nucl. Phys. B 289 (1987) 414.

[11] I. Antoniadis, C. Bachas, and C. Kounnas, Nucl. Phys. B 289 (1987) 87;

H. Kawai, D.C. Lewellen, and S.H.H. Tye, Nucl. Phys. B 288 (1987) 1.

[12] A.E. Faraggi and D.V. Nanopoulos, Phys. Rev. D 48 (1993) 3288;

A.E. Faraggi, Int. J. Mod. Phys. A 14 (1999) 1663.

[13] G.B. Cleaver, A.E. Faraggi and C. Savage, Phys. Rev. D 63 (2001) 066001;

G.B. Cleaver, D.J. Clements and A.E. Faraggi, Phys. Rev. D 65 (2002) 106003.

[14] K. Narain, Phys. Lett. B 169 (1986) 41;

K.S. Narain, M.H. Sarmadi and E. Witten, Nucl. Phys. B 279 (1987) 369.

[15] See e.g. H.P. Nilles, S. Ramos-Sanchez, M. Ratz and P.K.S. Vaudrevange, Eur. Phys. Jour. C59 (2009) 249;

F. Ploger, S. Ramos-Sanchez, M. Ratz and P.K.S. Vaudrevange, JHEP 0704, (2007) 063 . 
[16] K.S. Choi, S. Groot-Nibbelink, and M. Trapletti, JHEP 0412, (2004) 063. H.P. Nilles, S. Ramos-Sanchez, P.K.S. Vaudrevange and A. Wingerter, JHEP 0604, (2006) 050;

S. Ramos-Sanchez, Fortsch.Phys. 10 (2009) 907.

[17] C. Angelantonj and A. Sagnotti, Phys. Rep. 371 (2002) 1; [Erratum-ibid. 376 (2003) 339].

[18] C. Vafa and E. Witten, Nucl.Phys.Proc.Suppl. 46 (1996) 225;

C. Angelantonj, I. Antoniadis and K. Förger, Nucl. Phys. B 555 (1999) 116;

C. Angelantonj, M. Cardella and N. Irges, Phys. Lett. B 641 (2006) 472.

[19] H. Itoyama and T.R. Taylor, Phys. Lett. B 186 (1987) 129.

[20] J.D. Blum and K.R. Dienes, Nucl. Phys. B 516 (1998) 83; Phys. Lett. B 414 (1997) 260.

[21] A.E. Faraggi and M. Tsulaia, Eur. Phys. Jour. C54 (2008) 495.

[22] E.J. Martinec, D. Robbins and S. Sethi, arXiv:0904.3498. 